Voix et Images

voixetimages

\title{
La poésie de Paul-Marie Lapointe
}

\section{Jean Fisette}

Volume 13, numéro 1 (37), automne 1987

\section{Suzanne Lamy}

URI : https://id.erudit.org/iderudit/200695ar

DOI : https://doi.org/10.7202/200695ar

Aller au sommaire du numéro

Éditeur(s)

Université du Québec à Montréal

ISSN

0318-9201 (imprimé)

1705-933X (numérique)

Découvrir la revue

Citer cet article

Fisette, J. (1987). La poésie de Paul-Marie Lapointe. Voix et Images, 13(1),

174-178. https://doi.org/10.7202/200695ar

Ce document est protégé par la loi sur le droit d'auteur. L'utilisation des services d'Érudit (y compris la reproduction) est assujettie à sa politique d'utilisation que vous pouvez consulter en ligne.

https://apropos.erudit.org/fr/usagers/politique-dutilisation/
Cet article est diffusé et préservé par Érudit.

Érudit est un consortium interuniversitaire sans but lucratif composé de l'Université de Montréal, l'Université Laval et l'Université du Québec à Montréal. Il a pour mission la promotion et la valorisation de la recherche. https://www.erudit.org/fr/ 


\section{La poésie de Paul-Marie Lapointe}

\section{par Jean Fisette, Université du Québec à Montréal}

La prestigieuse collection «Pò̀tes d'aujourd'hui» publie enfin un Paul-Marie Lapointel dont la rédaction a été confiée à un fervent lecteur de poésie: Robert Melançon. La décision fut bonne, l'ouvrage est tout à fait satisfaisant.

Suivant les règles de la collection, on trouve un long texte de présentation suivi d'une anthologie dont la composition illustre, de façon exhaustive les divers aspects qu'a prises la poésie de Lapointe depuis le premier recueil de 1948 jusqu'à aujourd'hui.

Fidèle aux règles de la collection, Melançon, dans son texte de présentation intitulé «Dans la liberté première», procède à une description des recueils. Il en suit l'ordre de parution en s'intéressant tant aux aspects thématiques qu'aux 
questions théoriques touchées par la poésie de Lapointe. Or, le poète ayant inscrit sa démarche dans la modernité de l'écriture, il s'ensuit que ce texte de Melançon constitue aussi une introduction à la modernité de la poésie, sujet difficile s'il en est. Enfin, deux chapitres sont consacrés à des thématiques particulières, soit l'angoisse et l'amour.

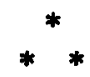

Dès le départ, la question de la modemité de la poésie est posée: contre l'usage quotidien, instrumental de la langue, la poésie se veut une aventure de langage, par et dans la langue: à cet égard, Melançon a eu la sagacité de présenter une longue citation de ce même Novalis auquel Lapointe empruntait le titre de l'édition rétrospective de 1968. Pourtant Melançon termine sur un énoncé qui me paraît présenter un certain risque: Le geste inaugural de la poésie, et son paradoxe, c'est le refus du sens... (p. 12). Certes, dans ce passage de l'introduction il faut lire sous le mot «sens» l'acception de «sens commun», stéréotypes, banalités et alors on ne peut qu'appuyer cette position; pourtant, tout au long de l'essai, cette même phrase sera reprise suivant une transformation insensible du mot «sens», de sorte qu'en conclusion, Melançon, traitant d'écRiturEs, proposera le terme d'abolition du sens, affirmant que Lapointe est resté fidèle à lui-même. Il y a là, me semble-t-il, une réelle difficulté, convaincu que je suis qu'écRiturEs marque une nette rupture dans la transformation de la poésie de Paul-Marie Lapointe. Nous y reviendrons plus bas.

Le Vierge incendié est présenté comme le texte de la révolte adolescente projetant des invectives contre la culture, la bienséance... (p. 18-19). Je lis cette proposition qui me paraît assez fine: Le texte se réduit d̀ une juxtaposition de signes dont l'assemblage, s'il n'exclut pas le sens, le reporte indéfiniment (p. 20); cependant, encore ici, un autre pas est franchi qui me laisse songeur: Dans quelques pages du Vierge incendié, cette ambition se retourne en asémie (p. 20). Si le texte du Vierge incendié se retourn[ait] en asémie, je me demande comment on pourrait parler de «révolte» et d' «invectives». Il semble encore ici que la terminologie manque de rigueur, le terme «asémie» étant nettement exagéré2.

Melançon termine sur ces quelques traits qui me semblent caractériser au mieux cette poésie de l'époque automatiste: un ton détaché, à la fois ironique et serein, un ton qui aussi, tient du jeu et de la fête (p. 22).

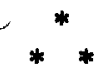


La suite poétique Arbres fournit l'occasion d'introduire la question des rapports entre la composition du poème et les formes musicales: ... cet ordre sans raideur, cette nécessité sans contrainte, cette liberté sans éparpillement (p. 31). La signification générale de cette suite poétique est ramenée à une tentative de réconciliation avec le monde (p. 32), suite aux témoignages de mauvaise vie et aux marques de rupture dont faisaient état les deux premiers recueils. Faut-il préciser que cette lecture s'inscrit contre les approches nationalistes qui souvent, surtout à l'époque de la parution en 1960, rattachaient ce texte aux renvendications socio-politiques alors si pressantes.

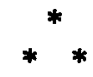

Dans les deux chapitres consacrés à Choix de poèmes et à Pour les âmes, la démarche poétique est donnée comme apprivoisement du monde (p. 34). Formellement, le fil du texte est analysé minutieusement en termes de double processus de rupture et de continuité dans le déroulement discursif: si les ruptures sont moins brusques, moins "sauvages» que dans les premiers recueils, il n'en reste pas moins que le discours présente des ruptures, lieux d'«improvisations» sur le modèle du jazz qui évitent au texte de s'enfermer dans la tautologie des lieux communs, soit le discours public, médiatique et démagogique que le poète affirme constamment vouloir dénoncer. Ce point d'équilibre entre la rupture et la continuité, la tautologie et l'éclatement, expliquerait, selon Melançon, que la plupart des critiques et lecteurs de poésie modeme continuent de considérer ces deux recueils comme les plus parfaitement réussis, en faisant un classique exemplaire de la modernité en poésie.

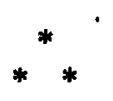

La thématique de l'amour, traitée au chapitre 9, constitue, à mon avis, le meilleur moment de l'essai: suivant une démarche critique rappelant celle de Meschonnic dans ses études sur Éluard, Melançon ramène la saisie du monde, à la fois physique et conceptuel, à une érotique généralisée: le terme de médiation (en fait un «thème» au sens strict de J.-P. Richard) renvoie à la pluralité, des choses, des sens: l'acte de discours consiste à multiplier les choses en les nommant, en multipliant les relations, en construisant, par le fait même, une représentation du cosmos comme ensemble plein, plein de sens, de relations, de saveurs, de chaleur. Et alors, les énumérations des parties du corps que l'on lisait dans le Vierge incendié (la jambe et le mollet et le genou et la cuisse...) sont ici réitérées, de sorte que les poèmes constituent autant de blasons de l'univers, comme en témoigne ce passage: L'acharnement sadien, la fureur et l'amertume d'un Bataille lui sont étrangers. L'érotisme prend chez lui la candeur d'une fête; il est une liberté plutôt qu'une libération. Les interdits n'ont pas dêtre transgressés, ils sont levés. Le corps - la chair dont Mallarmé disait qu'elle était triste - ne porte aucun poids de culpabilité (p. 59). Cette représentation de l'excès, du surplus, de l'extrême densité du monde n'est possible que dans la mesure où le 
discours brise la linéarité de l'énoncé, la «temporalité» de la phrase, pour devenir une saisie du monde où sens et formulation sont contemporains, constituent une même démarche. Acte poétique par excellence: «le réel absolu».

Les lectures de Tableau de l'amoureuse, Bouche rouge et du Tombeau de René Clavel conduisent à la question de fond soulevée par écRiturEs: Paul-Marie Lapointe l'affirmait lui-même: le poème ne veut plus rien dire.

Après avoir recensé quelques critiques parmi les plus autorisées qui d'une façon unanime ont rejeté ce monstre d'illisibilité (p. 80), Melançon affirme de façon nette l'effet de signature: si ce livre n'était signé par Paul-Marie Lapointe, en aurait-on parlé? L'aurait-on lu? Mieux encore, aurait-il été publié? Et Melançon d'inverser la question: c'est en raison de sa notoriêté même que PaulMarie Lapointe a pu ainsi se permettre de lancer ce pavé dans la mare de la création poétique en s'associant, lui le grand poète des années 1960-1970, aux poètes de la Nouvelle Barre du jour, voire en les dépassant sur leur propre terrain.

Mais alors, la position de Melançon paraît hésitante. D'une part, il traite de sa propre expérience de la lecture d'écRiturEs comme d'une activité d'ascèse (semblable à ce qu'a dû exiger la rédaction dudit texte), mais aussi comme d'une expérience d'apprentissage d'une lecture neuve, oubliant tous les réflexes, tous les mécanismes habituels de lecture, où la part de subjectivité du lecteur vient emplir les interstices du texte, lui insuffler de l'imaginaire; en ce sens écRiturEs paraît comme une réduction du poème, du discours, du texte (ces termes deviennent inutiles parce que non pertinents) au pur signifiant graphique: même la glose n'est plus permise.

Pourtant Melançon s'acharne malgré tout à trouver une spécificité, une raison à ce texte, faisant appel par exemple à ce passage de la Lettre volée où les signes recherchés échappent à l'observateur par leur «excessive évidence». Puis écRiturEs est donné comme une énorme rigolade du temps présent, un potlatch burlesque fait de rapprochements incongrus et de juxtapositions déplacées que ponctuent de grands éclats de rire (p. 85). Dans tous les cas, ces propositions restent trop générales pour apporter une réponse satisfaisante à la question.

Contre tout, Melançon maintient que ce livre possède une spécificité poétique qui excède le pur effet de signature alors que ce chapitre se termine sur cette simple proposition: Elle [cette oeuvre] est une énigme, qui nous renvoie d l'énigme du monde (p. 88).

De fait, écRiturEs a posé avec évidence la question probablement la plus fondamentale: si Bouche rouge et le Tombeau de René Clavel constituaient des 
oeuvres limites, il paraît qu'écRiturEs a transgressé cette limite, débordé les dernières conditions qui définissaient la modernité de la poésie (telle que la définissait Friedrich, par exemple); alors, où se situe ce livre? La distinction qu'utilisent les sémioticiens entre le SENS (comme effet spécifique d'un texte donné) et la SIGNIFICATION (comme la place que prend un objet donné dans son contexte ainsi que les relations qui le définissent) pourrait ici s'avérer utile: Bouche rouge, par exemple, s'inscrivait à l'intérieur des limites du SENS de sorte que la question de la SIGNIFICATION pouvait être éludée, mais, dans le cas d'écRiturEs, il n'y a plus d'expédient possible: ce qui est interrogé, c'est le statut même de la poésie parmi les autres catégories d'objets symboliques et de ce point de vue, le titre que Melançon a donné à ce chapitre («Mots croisés») est tout à fait significatif de l'envergure de la remise en cause. La pratique de lecture d'écRiturEs reste-t-elle le pur fait du miroitement d'un ecran paranoïaque auquel se référait Léonard? Et encore, de quelle lecture s'agit-il? Peut-être le défi à la linéarité sémantique de l'énoncé, qui fondait la poésie moderne, s'est-il transposé au niveau même de la présentation graphique? Peut-être, après tout, écRiturEs déborde-t-il tout simplement le seuil sémiotique pour ne reposer que sur la seule signification de l'objet garantie par la notoriété de l'auteur, à la façon de Duchamp qui plaçait un urinoir parmi les objets d'une exposition d'art? Ce qui est mis en cause ici, ne serait-ce pas un certain excès dans l'«environnementalisation» de l'art? Une chose me paraît cependant certaine: écRiturEs, comme les textes de Gauvreau à l'époque de la poésie pure, forcent l'interrogation sur les conditions de production du sens et de la signification. La question reste ouverte.

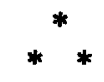

En somme, cet essai de Robert Melançon constitue une excellente introduction à la lecture de Paul-Marie Lapointe. Si l'on peut regretter l'insuffisance du traitement d'écRiturEs, d'une façon générale, la couverture du sujet est complète et pertinente.

1 Robert Melançon, Paul-Marie Lapolnte, Seghers, Paris, 1987, coll. «Poètes d'aujourd'hui». «Dans la liberté première», texte de présentation (p. 7-90), suivi d'une anthologie.

2 La célèbre formule de Reverdy: ... plus le rapport entre les objets sera éloigné et juste... est faussement attribuée à Breton qui l'avait citée dans le premier manifeste. 